\title{
The Prognostic Significance of Pre and Post Treatment Neutrophil to Lymphocyte Ratio in Breast Cancer Patients
}

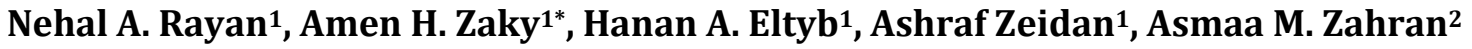 \\ ${ }^{1}$ Medical Oncology Department, South Egypt Cancer Institute, Assiut University, Assiut, Egypt \\ ${ }^{2}$ Clinical Pathology Department, South Egypt Cancer Institute, Assiut University, Assiut, Egypt \\ Email: *amenzaky74@yahoo.com
}

How to cite this paper: Rayan, N.A., Zaky, A.H., Eltyb, H.A., Zeidan, A. and Zahran, A.M. (2019) The Prognostic Significance of Pre and Post Treatment Neutrophil to Lymphocyte Ratio in Breast Cancer Patients. Journal of Cancer Therapy, 10, 525-536.

https://doi.org/10.4236/jct.2019.107044

Received: May 13, 2019

Accepted: July 9, 2019

Published: July 12, 2019

Copyright $\odot 2019$ by author(s) and Scientific Research Publishing Inc. This work is licensed under the Creative Commons Attribution International License (CC BY 4.0).

http://creativecommons.org/licenses/by/4.0/

\begin{abstract}
Background: The neutrophil/lymphocyte ratio (NLR) has been reported to reflect systemic inflammation and independent prognostic significance in different types of cancer. In present study, we analyzed the association between NLR and clinicopathologic features and verified the significance of NLR as a prognostic factor for patients with breast cancer. Patients and Methods: A total of 388 patients with stage I - III breast cancer were retrospectively recruited into this study. Associations with clinicopathologic factors and NLR were assessed; disease-free survival and overall survival were estimated. Results: There was no significant association between NLR and clinicopathologic factors. Patients with low pre/post-treatment NLR had longer OS. Conclusion: A high pre/postoperative NLR may be considered an important factor for predicting poor prognosis in non-metastatic breast cancer patients.
\end{abstract}

\section{Keywords}

Breast Cancer, NLR, DFS, OS

\section{Introduction}

Breast cancer is the most frequently diagnosed life-threatening cancer in women and the leading cause of cancer death among women [1]. In spite of recent advances in treatment of breast cancer, the prognosis remains unsatisfactory due to the recurrence and metastasis, with 5-year and 10-year survival rate of approximately $80 \%$ and $60 \%$ [2]. Different studies have reported tumor size, pathologic tumor, node, metastasis (TNM) staging, Ki67 expression, receptor status (estrogen receptor, ER; progesterone receptor, PR; human epidermal growth factor 
receptor 2, HER2) and molecular subtypes (luminal-type, HER2-positive, and triple-negative) are significantly associated with the prognosis of patients with BC [3]. Their prognostic accuracy is also reported to be unsatisfactory, with the example of the different TNM stage or molecular subtypes having the same prognosis [4]. Thus, more easily available and efficient preoperative prognostic parameters are desirable to guide individualized treatment. Therefore, it is essential to stratify the patients preoperatively.

Recent breakthroughs in cancer immunology substantiated that the host immune system correlates with cancer development and progression, and immunomodulating therapy has emerged as an effective novel therapeutic strategy [5]. Furthermore, the host immune system should be taken into account even during conventional chemotherapy treatment, as it has been found to influence the clinical response to chemotherapy [6]. Carcinogenesis and tumor growth are associated with chronic systemic inflammation, which is associated with tumor progression and subsequent poor outcomes [7]. Both lymphocytes and macrophages are key immune cells in the inflammatory response. They play an important role in tumor eradication via inhibition tumor cell proliferation, migration and inducing cytotoxic cell death [8].

Recent reports suggested that the peripheral blood-based parameters, such as absolute lymphocyte count (ALC), and neutrophil to lymphocyte ratio (NLR), are associated with host immunity response [9]. Moreover, there is a reliable correlation between the above parameters and increased survival time in a wide range of malignancies [10]. Lymphocyte infiltration into cancer tissue has been associated with a better prognosis in various malignancies [11]. There is increasing evidence that the NLR is associated with long-term outcomes, so this ratio has gained much interest, with several studies over the last 5 years investigating its role in predicting long-term outcomes in various cancer populations, including lung, colorectal, stomach, liver, and pancreatic cancer. Based on studies that show the association between high NLR and increased mortality in breast cancer, some studies suggest that NLR is an important factor predicting the response to neoadjuvant chemotherapy in breast cancer patients [12].

\section{Patients and Methods}

Retrospective study included 388 patients with breast cancer, stage I-III were treated between 2009 and 2015 at South Egypt Cancer Institute, Assiut University. The study was approved by Institutional Review Board (IRB) of South Egypt Cancer Institute. Patient medical records were reviewed; Tumor stage, $\mathrm{T}$ and $\mathrm{N}$ factors were stratified based on the TNM Classification of Malignant Tumors, UICC Seventh Edition [13]. Tumors were classified into subtypes according to the immunohistochemical expression of estrogen receptor, progesterone receptor and HER2. Overall survival (OS) time was the period from the time of diagnosis to the time of death from any cause. Disease-free survival (DFS) was defined as freedom from all local, loco-regional, and distant recurrences. All pa- 
tients were followed up by physical examination every 3 months, ultrasonography every 6 months, and computed tomography and bone scintigraphy annually.

Patients who had inflammatory, metastatic, or pregnancy-related breast cancer'; Patients with systemic inflammatory diseases, such as systemic lupus erythematosus, and rheumatoid arthritis; Patients with chronic diseases (liver cirrhosis, or end-stage renal disease) all were excluded.

Pre-treatment and post-treatment peripheral blood based parameters (total WBC count, ALC, ANC, hemoglobin level, and platelets count), were collected. For pre-treatment peripheral blood parameters, blood samples were collected at the time of diagnosis before neoadjuvant chemotherapy or surgery. NLR was calculated by dividing ANC by ALC. For post-treatment peripheral blood parameters, blood samples were collected 3 weeks after surgery. Receiver operating curve (ROC) analysis was performed to select the most appropriate cutoff values for pre-and post-treatment NLR in order to stratify patients at a high risk of cancer recurrence or death.

\section{Result}

This study was conducted retrospectively on (388) patients with breast cancer from January 2009 to December 2015. Patients' characteristics at diagnosis are shown in Table 1. As regarding pre-treatment hematological data, the mean absolute neutrophil count (ANC) was $4.18 \pm 0.1 \times 109$ cells/L, mean absolute lymphocyte count (ALC) was $2.25 \pm 0.04 \times 109$ cells/L, and mean NLR was $2.09 \pm$ 0.7. Post-treatment hematological data, the mean ANC was $3.67 \pm 0.1 \times 109$ cells/L, mean ALC was $1.91 \pm 0.04 \times 109$ cells/L, mean NLR was $2.21 \pm 0.1$. As shown in Table 2.

No significant change NLR ration related to age.

Table 1. Clinic pathological feature of 388 breast cancer patient.

\begin{tabular}{lc}
\hline \multicolumn{1}{c}{ Age in years (mean \pm SD) } & $47.23 \pm 10.1$ \\
\hline Menopausal Status: & $192(49.5 \%)$ \\
- Pre-menopausal & $196(50.5 \%)$ \\
- Post-menopausal & \\
Pathology & $350(90.2 \%)$ \\
- IDC & $18(4.6 \%)$ \\
- ILC & $14(3.6 \%)$ \\
- Mixed & $2(0.5 \%)$ \\
- DCIS & $3(1.1 \%)$ \\
- Others & \\
Histological Grade: & $5(1.4 \%)$ \\
- Grade I & $344(88.6 \%)$ \\
- Grade II & $39(10 \%)$ \\
- Grade III &
\end{tabular}


Continued

T-Stage:

- $\mathrm{T} 1$

$32(8.2 \%)$

- $\mathrm{T} 2$

$216(55.6 \%)$

- $\mathrm{T} 3$

$73(18.8 \%)$

- T4

$44(11.3 \%)$

- $\mathrm{Tx}$

$23(6.1 \%)$

Nodal-Stage

- No

$116(29.9 \%)$

- N1

$127(32.7 \%)$

- N2

$62(15.9 \%)$

- N3

$65(16.7 \%)$

- $\mathrm{Nx}$

$18(4.8 \%)$

Hormone Receptor Status:

- ER PR Positive

$201(51.8 \%)$

- ER Positive PR Negative

$49(12.6 \%)$

- ER Negative PR Positive

$11(2.9 \%)$

- ER PR Negative

$127(32.7 \%)$

- Triple Negative

$28(7.2 \%)$

HER2-neu expression:

- $0 /+1$

$110(28.4 \%)$

$-+2$

$20(4.9 \%)$

- +3

$38(9.8 \%)$

- Unknown

$221(56.9 \%)$

Table 2. Hematological data analysis (pre-treatment and post-treatment).

\begin{tabular}{cccc}
\hline Parameter & Pre-treatment & Post-treatment & P-value $^{*}$ \\
\hline ANC & $4.18 \pm 0.1$ & $3.67 \pm 0.1$ & $<0.001$ \\
ALC & $2.25 \pm 0.04$ & $1.91 \pm 0.04$ & $<0.001$ \\
NLR & $2.09 \pm 0.7$ & $2.21 \pm 0.1$ & $=0.274$ \\
\hline
\end{tabular}

\subsection{Diagnostic Performance of Pre-Treatment and Post-Treatment NLR in Survival Prediction}

It was noticed that at cutoff point $\leq 2.57$, pretreatment NLR had $81 \%$ sensitivity and $33 \%$ specificity for prediction of survival in all studied patients with area under the curve was 0.54 and $\mathrm{P}$ value was 0.01 (Table 3 ). It was noticed that at cutoff point $\leq 2.27$, post-treatment NLR had $71 \%$ sensitivity and $48 \%$ specificity for prediction of survival in all studied patients with area under the curve was 0.59 and $\mathrm{P}$ value was $<0.001$ (Table 3 ). 
Table 3. Cut-off points for NLR.

\begin{tabular}{cccc}
\hline Indices & Pre-treatment & Post-treatment & P value* $^{*}$ \\
\hline NLR & $\leq 2.57$ & $\leq 2.27$ & $<0.001$ \\
\hline
\end{tabular}

$\mathrm{P}$ value was significant if $<0.05 .{ }^{*} \mathrm{Mc}-\mathrm{Nemar}$ test was used to compare the percentages between groups Prevs. Post-treatment $\%$ was calculated from the total number. NLR, neutrophil to lymphocyte ratio.

\subsection{Characteristics of the Patients Based on Cutoff Point of Pre-Treatment NLR}

Of the total patients (388), 316 patients had pre-treatment NLR less than 2.57 and 72 patients had pre-treatment NLR more than 2.57. As shown in Table 4, based on cutoff point of pre-treatment NLR; it was noticed that there were no statistical significant differences between those patients with pre-treatment NLR $<2.57$ and those with pre-treatment NLR $\geq 2.57$ as regarding demographic data, baseline laboratory data, tumor characteristics, adjuvant radiotherapy and chemotherapy.

\subsection{Outcome of Studied Patients Based on Cutoff Point of Pre-Treatment NLR}

Frequency of relapse was higher in patients with pre-treatment NLR $\geq 2.57$ $(36.1 \%$ vs. $26.3 \%)$ while those with pre-treatment $\mathrm{NLR}<2.57$ had higher frequency of survival ( $82 \%$ vs. $68 \%$ ). Patients with pre-treatment NLR $<2.57$ had longer DFS than patients with pre-treatment NLR $\geq 2.57$ (83 months vs. 73 months) but with no statistically significant p-value (0.122). As regarding overall survival, patients with pre-treatment NLR $<2.57$ had longer OS than patients with pre-treatment NLR $\geq 2.57$ (93 months vs. 81 months) with log-rank p-value 0.016 between the two groups (Figure $1 \&$ Figure 2).

\subsection{Outcome of Studied Patients Based on Cuttoff Point of Post-Treatment NLR}

Patients with post-treatment NLR $<2.27$ had longer DFS than patients with post-treatment NLR $\geq 2.27$ (83 months vs. 75 months) but with no statistically significant p-value (0.091). As regarding overall survival, Patients with post-treatment NLR $<2.27$ had longer OS than patients with post-treatment NLR $\geq 2.27$ (94 months vs. 80 months) with log-rank p-value 0.011 between the two groups (Figure 3 \& Figure 4).

Survival analysis of all studied breast cancer patients summarized in Table 5.

\section{Discussion}

Breast cancer is the most frequent malignancy in women, being one of the main causes of death from cancer. Although its incidence has increased, mortality has decreased in the last few decades, which can be attributed to an improvement in early diagnosis and treatment [14]. For patients in our study, the median OS was 60 months with range (62 - 65 months), the median DFS was 54 months with 
Table 4. Demographic data of patients based on cutoff point of pre-treatment NLR.

\begin{tabular}{|c|c|c|c|}
\hline \multirow{2}{*}{ Variables } & \multicolumn{2}{|c|}{ Pre-treatment NLR } & \multirow{2}{*}{$P$ value } \\
\hline & $<2.57(n=316)$ & $\geq 2.57(n=72)$ & \\
\hline Age (years) & $47.34 \pm 10.03$ & $46.85 \pm 10.45$ & 0.49 \\
\hline \multicolumn{4}{|l|}{ Menopausal status: } \\
\hline - Pre-menopausal & $154(48.8 \%)$ & $37(51.8 \%)$ & 0.36 \\
\hline - Post-menopausal & $162(51.2 \%)$ & $35(48.2 \%)$ & \\
\hline \multicolumn{4}{|l|}{ Pathological type: } \\
\hline - IDC & $285(90.1 \%)$ & $65(90.6 \%)$ & \\
\hline - ILC & $15(4.6 \%)$ & $3(4.7 \%)$ & \\
\hline - Mixed & $4(1.3 \%)$ & 0 & 0.72 \\
\hline - DCIS & $1(0.3 \%)$ & $1(1.2 \%)$ & \\
\hline - Others & $11(3.6 \%)$ & $3(3.5 \%)$ & \\
\hline \multicolumn{4}{|l|}{ Histological grade: } \\
\hline - Grade I & $4(1.3 \%)$ & $1(1.2 \%)$ & 006 \\
\hline - Grade II & $268(88.4 \%)$ & $76(89.4 \%)$ & 0.06 \\
\hline - Grade III & $31(10.2 \%)$ & $8(9.5 \%)$ & \\
\hline \multicolumn{4}{|l|}{ Tumor stage: } \\
\hline - $\mathrm{T} 1$ & $27(8.3 \%)$ & $6(8.2 \%)$ & \\
\hline - $\mathrm{T} 2$ & $181(57.4 \%)$ & $35(49.4 \%)$ & 0.33 \\
\hline - $\mathrm{T} 3$ & $59(18.8 \%)$ & $14(18.8 \%)$ & 0.33 \\
\hline - $\mathrm{T} 4$ & $30(9.6 \%)$ & $12(17.6 \%)$ & \\
\hline - $\mathrm{Tx}$ & $19(5.9 \%)$ & $5(5.9 \%)$ & \\
\hline \multicolumn{4}{|l|}{ Nodal stage: } \\
\hline - N0 & $95(30 \%)$ & $21(29.4 \%)$ & \\
\hline - N1 & $106(33.7 \%)$ & $21(29.4 \%)$ & \\
\hline - $\mathrm{N} 2$ & $53(16.8 \%)$ & $9(12.9 \%)$ & 0.49 \\
\hline - $\mathrm{N} 3$ & $48(15.2 \%)$ & $16(22.4 \%)$ & \\
\hline - $\mathrm{Nx}$ & $14(4.3 \%)$ & $5(5.9 \%)$ & \\
\hline \multicolumn{4}{|l|}{ Hormone receptor status: } \\
\hline - ER/PR positive & $163(51.4 \%)$ & $38(52.9 \%)$ & \\
\hline - ER positive and PR negative & $42(13.2 \%)$ & $7(10.6 \%)$ & 0.09 \\
\hline - ER negative and PR positive & $9(2.9 \%)$ & $2(2.4 \%)$ & \\
\hline - ER/PR negative & $102(32.2 \%)$ & $25(34.1 \%)$ & \\
\hline \multicolumn{4}{|l|}{ HER-2 neu expression (by IHC): } \\
\hline - $0 /+1$ & $87(27.4 \%)$ & $22(30.6 \%)$ & \\
\hline$\bullet+2$ & $18(5.3 \%)$ & $4(4.7 \%)$ & 0.17 \\
\hline - +3 & $35(11.6 \%)$ & $2(3.5 \%)$ & \\
\hline - Unknown & $176(55.8 \%)$ & $44(61.2 \%)$ & \\
\hline Triple negative: & $22(7 \%)$ & $8(10.6 \%)$ & 0.21 \\
\hline
\end{tabular}

Data was expressed in form of mean (SD), frequency (percentage). P value was significant if $<0.05$. NLR, neutrophil to lymphocyte ratio; ER, estrogen receptor; PR, progesterone receptor, HER2: Human epidermal growth factor receptor 2; IHC: Immunohistochemistry.

Table 5. Survival analysis of breast cancer patients.

\begin{tabular}{lc}
\hline \multicolumn{2}{c}{ Relapse (Local \& Distant) } \\
\hline - Yes & $109(28 \%)$ \\
- No & $277(71.4 \%)$ \\
- Secondary malignancy & $2(0.6 \%)$ \\
\hline
\end{tabular}




\section{Continued}

\begin{tabular}{lc}
\hline \multicolumn{2}{c}{ Death Status } \\
\hline - Alive & $299(77 \%)$ \\
- Dead & $89(23 \%)$ \\
Median overall survival in months (95\%-CI) & $60(62-65)$ \\
Median disease-free survival in months (95\%-CI) & $54(47-60)$ \\
\hline
\end{tabular}

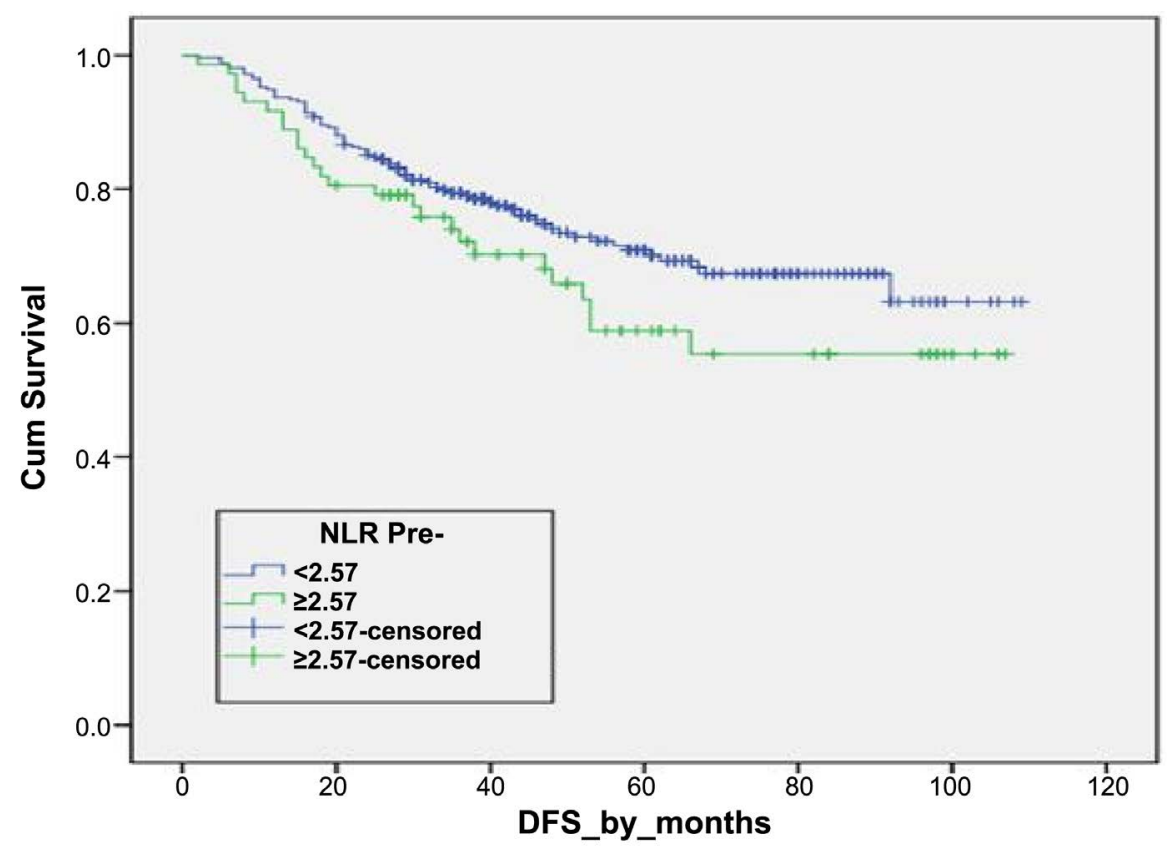

Figure 1. Disease free survival based on pretreatment NLR in the study.

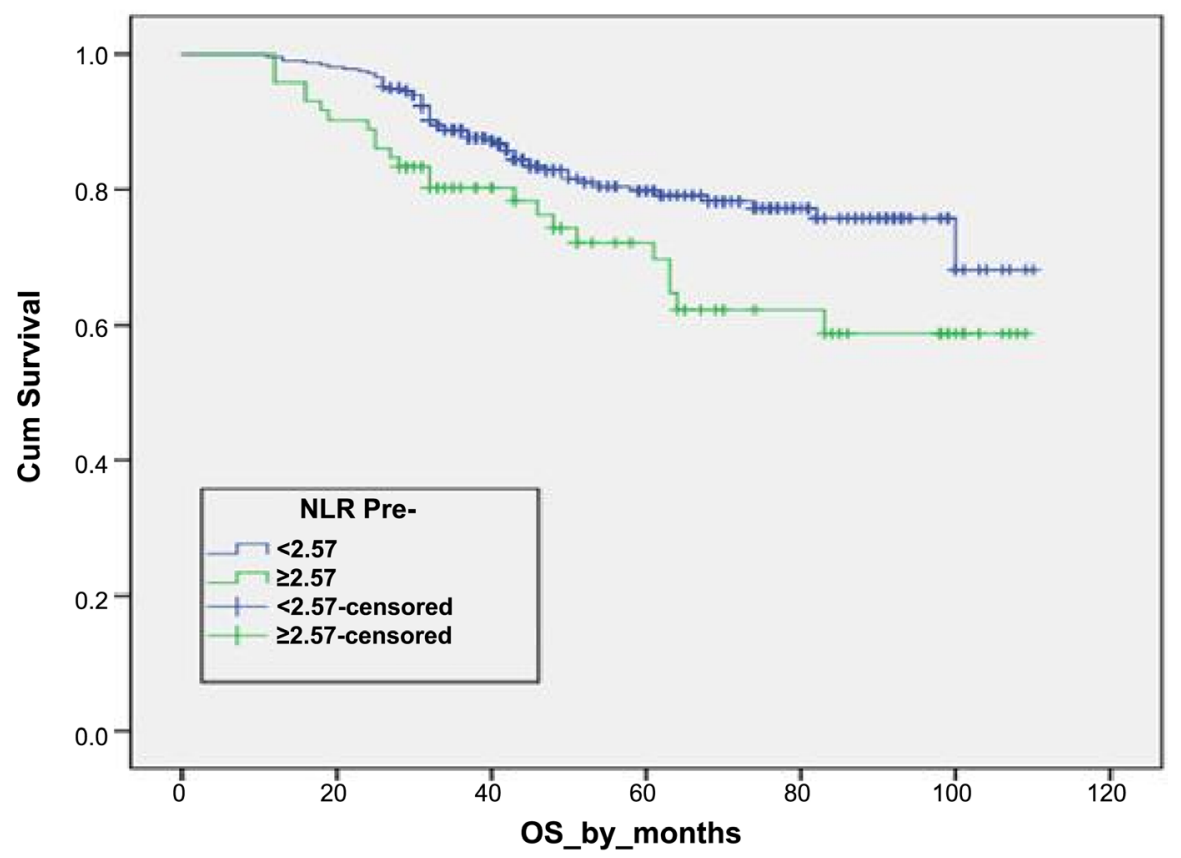

Figure 2. Overall survival in patients based on pretreatment NLR. 


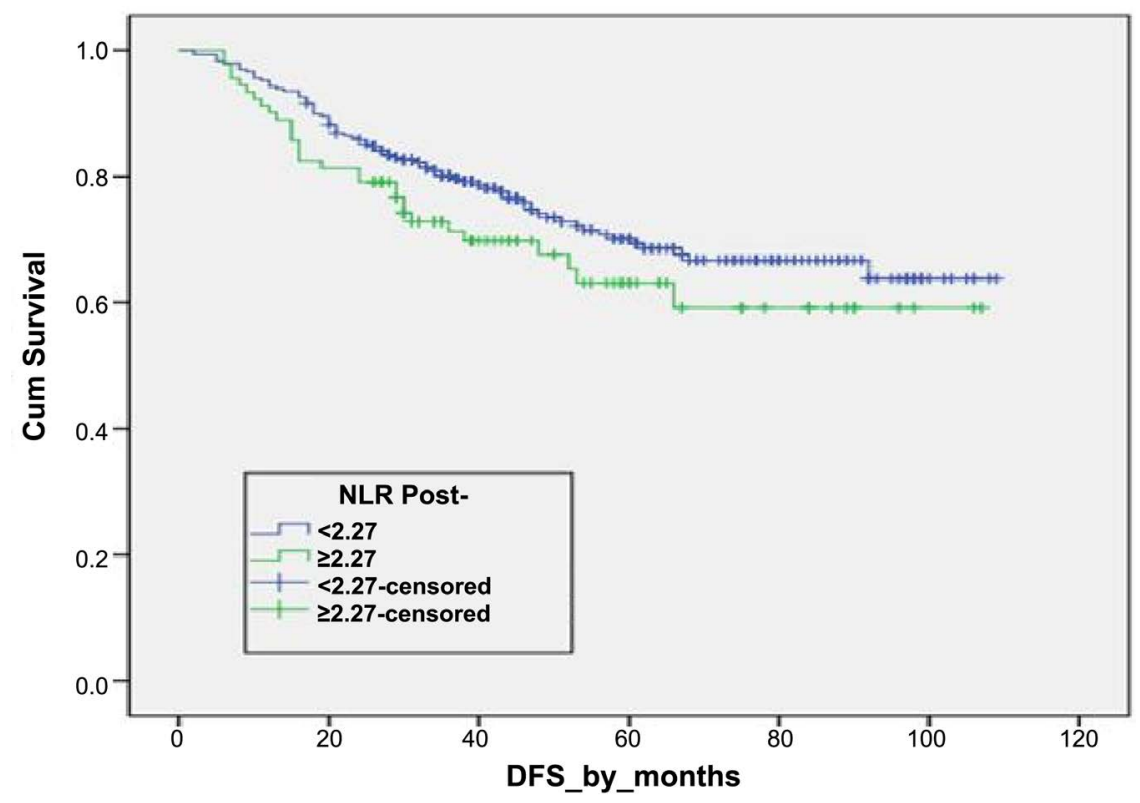

Figure 3. Disease free survival in all enrolled patients based on post-treatment NLR.

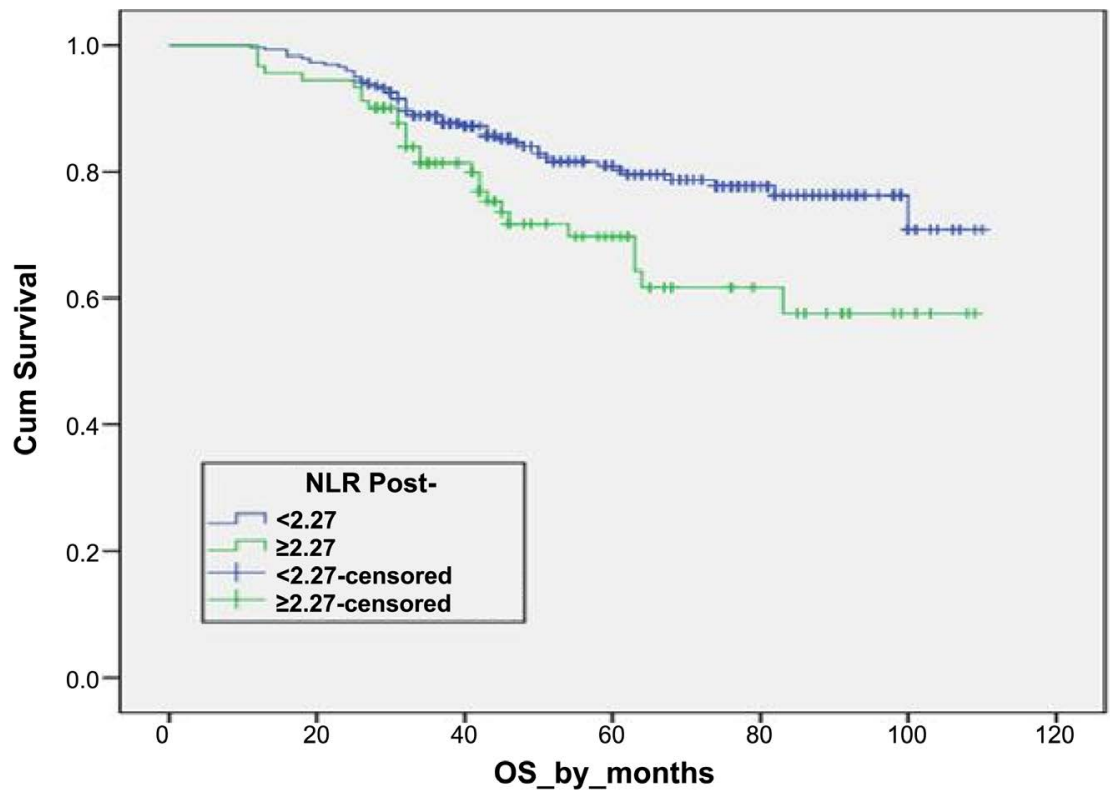

Figure 4. Overall survival in patients based on post-treatment NLR.

range (47 - 60 months). The median DFS time was 54 months. Neutrophil to lymphocyte ratio (NLR) has been analyzed in connection with several types of cancer including lung cancer [15], gastric cancer [16], colorectal cancer [17], pancreatic cancer [18], hepatocellular carcinoma [8], ovarian cancer [19], and some hematological malignancies [20]. And low NLR ratios have been associated with high DFS, OS and a better prognosis in many different cancer patients [21]. In this study, we analyzed a cohort of 388 patients with non-metastatic breast cancer to demonstrate that the NLR could predict prognosis in these patients. For patients in our study, the cutoff value of pretreatment NLR was 2.57. Most 
studies that evaluated NLR in breast cancer detected NLR with the range of (1.9 4.0) [22].

In the present study, we evaluated the relationship between pretreatment NLR, DFS, and OS for with non-metastatic breast cancer patients. We found that high pretreatment NLR is associated with increase rate of relapse $36.1 \%$ for patients with high pretreatment NLR vs. $26.3 \%$ in patients with low pretreatment NLR) and lower DFS (83 months for patients with low pretreatment NLR vs. 73 months for patients with high pretreatment NLR) but without statistical significance. As regarding OS there was statistical significance between the two groups (93 months for patients with low pretreatment NLR vs. 81 months for patients with high pretreatment NLR) (log-rank p-value $=0.016)$. These findings are in agreement with the results of [23] (Ren et al. 2018) and [24] (Das 2017). However, in contrast to our results, Ren 2018 evaluated TNBC patients and Das 2017 results showed significant relation between NLR and DFS.

As regarding correlation between NLR, tumor stage and hormonal receptor status, our study showed that high pretreatment NLR had no correlation with the tumor staging $(\mathrm{p}=0.33)$, nodal staging $(\mathrm{p}=0.49)$, or hormonal receptor status $(p=0.09)$ and HER2-neu status $(p=0.17)$. These results were in agreement with the results of [25] and [26] but in contrast to the results of (Das et al.) who showed significant correlation between pre-treatment NLR and tumor size ( $\mathrm{p}=0.017)$ [24] and [27] (Azab et al.) who showed significant correlation between pre-treatment NLR and nodal status $(\mathrm{p}=0.005)$, ER positive tumor $(\mathrm{p}=$ $0.02)$, $P R$ positive tumor $(\mathrm{p}=0.04)$ and tumor staging $(\mathrm{p}<0.001)$.

Here, in present study, we evaluated the cutoff for post-treatment NLR in the studied patients. There was statistical significance between both groups and OS (94 months for patients with low pretreatment NLR vs. 80 months in patients with high pretreatment NLR), but again there was no significant correlation between post-treatment NLR and any of the following: DFS, tumor staging, nodal staging, and hormonal and HER2-neu status. To the best of our knowledge, no studies have evaluated the role of post-treatment NLR in predicting response and prognosis in non-metastatic breast cancer patients, but a study by [28] (Chowdhary et al.) which examined the significance of post-treatment NLR but in brain metastasis treated with stereotactic radiosurgery and found that post-treatment NLR is inversely associated with OS in patients with brain metastasis.

\section{Conclusion}

NLR is a simple marker of sub-clinical inflammation that can be easily assessed using white blood cell counting. Interestingly, a high pre/postoperative NLR may be considered an important factor for predicting poor prognosis in non-metastatic breast cancer patients. We need multicenter study to avoid heterogenicity.

\section{Conflicts of Interest}

The authors declare no conflicts of interest regarding the publication of this paper. 


\section{References}

[1] Chalasani, P. (2018) Breast Cancer.

[2] Maishman, T., Cutress, R.I., Hernandez, A., et al. (2017) Local Recurrence and Breast Oncological Surgery in Young Women with Breast Cancer: The POSH Observational Cohort Study. Annals of Surgery, 266, 165-172.

https://doi.org/10.1097/SLA.0000000000001930

[3] Hadad, S.M., Jordan, L.B., Roy, P.G., et al. (2016) A Prospective Comparison of ER, PR Ki67 and Gene Expression in Paired Sequential Core Biopsies of Primary, Untreated Breast Cancer. BMC Cancer, 16, 745. https://doi.org/10.1186/s12885-016-2788-x

[4] Engstrøm, M.J., Opdahl, S., Hagen, A.I., et al. (2013) Molecular Subtypes, Histopathological Grade and Survival in a Historic Cohort of Breast Cancer Patients. Breast Cancer Research and Treatment, 140, 463-473. https://doi.org/10.1007/s10549-013-2647-2

[5] Stromnes, I.M., Greenberg, P.D. and Hingorani, S.R. (2014) Molecular Pathways: Myeloid Complicity in Cancer. Clinical Cancer Research, 20, 5157-5170. https://doi.org/10.1158/1078-0432.CCR-13-0866

[6] Ji, H., Xuan, Q., Yan, C., et al. (2016) The Prognostic and Predictive Value of the Lymphocyte to Monocyte Ratio in Luminal-Type Breast Cancer Patients Treated with CEF Chemotherapy. Oncotarget, 7, 34881-34889.

https://doi.org/10.18632/oncotarget.8993

[7] Diakos, C.I., Charles, K.A., McMillan, D.C., et al. (2014) Cancer-Related Inflammation and Treatment Effectiveness. The Lancet Oncology, 15, e493-e503. https://doi.org/10.1016/S1470-2045(14)70263-3

[8] Motomura, T., Shirabe, K., Mano, Y., et al. (2013) Neutrophil-Lymphocyte Ratio Reflects Hepatocellular Carcinoma Recurrence after Liver Transplantation via Inflammatory Microenvironment. Journal of Hepatology, 58, 58-64.

https://doi.org/10.1016/j.jhep.2012.08.017

[9] Jiang, L., Zhao, Z., Jiang, S., et al. (2015) Immunological Markers Predict the Prognosis of Patients with Squamous Non-Small Cell Lung Cancer. Immunologic Research, 62, 316-324. https://doi.org/10.1007/s12026-015-8662-0

[10] Cihan, Y.B., Arslan, A., Cetindag, M.F., et al. (2014) Lack of Prognostic Value of Blood Parameters in Patients Receiving Adjuvant Radiotherapy for Breast Cancer. Asian Pacific Journal of Cancer Prevention, 15, 4225-4231. https://doi.org/10.7314/APJCP.2014.15.10.4225

[11] De Giorgi, U., Mego, M., Scarpi, E., et al. (2012) Relationship between Lymphocytopenia and Circulating Tumor Cells as Prognostic Factors for Overall Survival in Metastatic Breast Cancer. Clinical Breast Cancer, 12, 264-269. https://doi.org/10.1016/j.clbc.2012.04.004

[12] Chen, Y., Chen, K., Xiao, X., et al. (2016) Pretreatment Neutrophil-to-Lymphocyte Ratio Is Correlated with Response to Neoadjuvant Chemotherapy as an Independent Prognostic Indicator in Breast Cancer Patients: A Retrospective Study. BMC Cancer, 16, 320. https://doi.org/10.1186/s12885-016-2352-8

[13] American Joint Committee on Cancer Breast, Amin, M.B., Edge, S., Greene, F., Byrd, D.R., Brookland, R.K., et al. (2017) AJCC Cancer Staging Manual. 8th Edition, Springer, New York.

[14] Miller, K.D., Siegel, R.L., Lin, C.C., et al. (2016) Cancer Treatment and Survivorship Statistics, 2016. CA: A Cancer Journal for Clinicians, 66, 271-289. 
https://doi.org/10.3322/caac.21349

[15] Tomita, M., Shimizu, T., Ayabe, T., et al. (2012) Elevated Preoperative Inflammatory Markers Based on Neutrophil-to-Lymphocyte Ratio and C-Reactive Protein Predict Poor Survival in Resected Non-Small Cell Lung Cancer. Anticancer Research, 32, 3535-3538. https://doi.org/10.1155/2012/463520

[16] Hwang, G.Y., Baek, D.W., Cho, H.J., et al. (2018) Elevated Neutrophil-to-Lymphocyte Ratio Predicts Survival in Patients with Advanced Gastric Cancer Treated with Trastuzumab Combination Chemotherapy. Anticancer Research, 38, 3151-3156. https://doi.org/10.21873/anticanres.12578

[17] Mallappa, S., Sinha, A., Gupta, S., et al. (2013) Preoperative Neutrophil to Lymphocyte Ratio $>5$ Is a Prognostic Factor for Recurrent Colorectal Cancer. Colorectal Disease, 15, 323-328. https://doi.org/10.1111/codi.12008

[18] Bhatti, I., Peacock, O., Lloyd, G., et al. (2010) Preoperative Hematologic Markers as Independent Predictors of Prognosis in Resected Pancreatic Ductal Adenocarcinoma: Neutrophil-Lymphocyte versus Platelet-Lymphocyte Ratio. The American Journal of Surgery, 200, 197-203. https://doi.org/10.1016/j.amjsurg.2009.08.041

[19] Chen, S., Zhang, L., Yan, G., et al. (2017) Neutrophil-to-Lymphocyte Ratio Is a Potential Prognostic Biomarker in Patients with Ovarian Cancer: A Meta-Analysis. BioMed Research International, 2017, Article ID: 7943467. https://doi.org/10.1155/2017/7943467

[20] Wang, J., Zhou, X., Liu, Y., et al. (2017) Prognostic Significance of Neutrophil-to-Lymphocyte Ratio in Diffuse Large B-Cell Lymphoma: A Meta-Analysis. PLoS ONE, 12, e0176008. https://doi.org/10.1371/journal.pone.0176008

[21] Ethier, J.L., Desautels, D., Templeton, A., et al. (2017) Prognostic Role of Neutrophil-to-Lymphocyte Ratio in Breast Cancer: A Systematic Review and Meta-Analysis. Breast Cancer Research, 19, 2. https://doi.org/10.1186/s13058-016-0794-1

[22] Ren, K., Yin, Y., He, F., et al. (2018) Prognostic Role of Derived Neutrophil-to-Lymphocyte Ratio in Surgical Triple-Negative Breast Cancer. Cancer Management and Research, 10, 4891-4898. https://doi.org/10.2147/CMAR.S180695

[23] Das, C. (2017) Prognostic Significance of Derived Neutrophil-Lymphocyte Ratio in Non-Metastatic Breast Cancer. Advances in Human Biology, 7, 54-60. https://doi.org/10.4103/2321-8568.205387

[24] Guthrie, G.J., Charles, K.A., Roxburgh, C.S., et al. (2013) The Systemic Inflammation-Based Neutrophil-Lymphocyte Ratio: Experience in Patients with Cancer. Critical Reviews in Oncology/Hematology, 88, 218-230.

https://doi.org/10.1016/j.critrevonc.2013.03.010

[25] Ulas, A., Avci, N., Kos, T., et al. (2015) Are Neutrophil/Lymphocyte Ratio and Platelet/Lymphocyte Ratio Associated with Prognosis in Patients with HER2-Positive Early Breast Cancer Receiving Adjuvant Trastuzumab? Journal of Buon, 20, 714-722.

[26] Chowdhary, M., Switchenko, J.M., Press, R.H., et al. (2018) Post-Treatment Neutrophil-to-Lymphocyte Ratio Predicts for Overall Survival in Brain Metastases Treated with Stereotactic Radiosurgery. Journal of Neuro-Oncology, 139, 689-697. https://doi.org/10.1007/s11060-018-2914-5

[27] Azab, B., Shah, N., Radbel, J., et al. (2013) Pretreatment Neutrophil/Lymphocyte Ratio Is Superior to Platelet/Lymphocyte Ratio as a Predictor of Long-Term Mortal- 
ity in Breast Cancer Patients. Medical Oncology, 30, 432.

https://doi.org/10.1007/s12032-012-0432-4

[28] Elyasinia, F., Keramati, M.R., Ahmadi, F., et al. (2017) Neutrophil-Lymphocyte Ratio in Different Stages of Breast Cancer. Acta Medica Iranica, 55, 228-232. 